\title{
Erratum
}

Kardiologe 2019· 13:236

https://doi.org/10.1007/s12181-019-0337-y

Online publiziert: 11. Juli 2019

(c) Deutsche Gesellschaft für Kardiologie Herz- und Kreislaufforschung e.V. Published by Springer Medizin Verlag $\mathrm{GmbH}$, ein Teil von Springer Nature - all rights reserved 2019

C. Burgstahler $\cdot$ A. Pressler ${ }^{2,11} \cdot$ S. Berrisch-Rahmel ${ }^{3} \cdot$ K.-P. Mellwig ${ }^{4} \cdot$ C. Bongarth ${ }^{5} \cdot$ M. Halle ${ }^{2} \cdot$ J. Niebauer ${ }^{6} \cdot$ R. Hambrecht ${ }^{7} \cdot$ S. Gielen ${ }^{8} \cdot$ J. M. Steinacker ${ }^{9} \cdot$ J. Scharhag $^{10}$ ${ }^{1}$ Medizinische Klinik, Abteilung Sportmedizin, Universitätsklinikum Tübingen, Tübingen, Deutschland ${ }^{2}$ Lehrstuhl und Poliklinik für Präventive und Rehabilitative Sportmedizin, Klinikum rechts der Isar, TU München, München, Deutschland

${ }^{3}$ CardioCentrum Düsseldorf und Institut für Prävention und Sportmedizin, Düsseldorf, Deutschland

${ }^{4}$ Klinik für Allgemeine und Interventionelle Kardiologie/Angiologie, Schwerpunkt Sportkardiologie, Herzund Dialysezentrum NRW, Bad Oeynhausen, Deutschland

${ }^{5}$ Rehabilitationszentrum am Starnberger See, Klinik Höhenried gGmbH, Bernried, Deutschland ${ }^{6}$ Institut für Sportmedizin des Landes Salzburg, Sportmedizin des Olympiazentrums Salzburg-Rif, Universitätsinstitut für präventive und rehabilitative Sportmedizin, Salzburg, Österreich

${ }^{7}$ Kardiologie und Angiologie, Klinikum Links der Weser, Bremen, Deutschland

${ }^{8}$ Abteilung Kardiologie, Angiologie und Intensivmedizin, Klinikum Lippe, Detmold, Deutschland

${ }^{9}$ Medizinische Klinik, Abteilung Sport- und Rehabilitationsmedizin, Universitätsklinikum Ulm, Ulm, Deutschland

${ }^{10}$ Abteilung für Sportmedizin, Leistungsphysiologie und Prävention, Institut für Sportwissenschaft, Universität Wien, Wien, Österreich

${ }^{11}$ Privatpraxis für Kardiologie, Sportmedizin, Prävention \& Rehabilitation, München, Deutschland

\section{Erratum zu: Curriculum Sportkardiologie}

\section{Erratum zu:}

Kardiologe 2019

https://doi.org/10.1007/s12181-019-

0299-0

Im Abschnitt „Übergangsregelung“ des Beitrags wird als Voraussetzung für alle Stufen fälschlicherweise die Zusatzbezeichnung Sportmedizin gefordert. Diese ist jedoch lediglich für die Anerkennung der Stufe 3 Voraussetzung. Wir bitten um Beachtung des unten angefügten, korrigierten Abschnitts:

Fachärzte für Innere Medizin und Kardiologie, die für die vorangegangenen 3 Jahre vor Antragstellung die geforderten Inhalte (s. Abschnitt „Praktische Durchführung der Qualifizierung “) und Mindestzahlen in der Sportkardiologie nachweisen, können auf Antrag im Rahmen einer Übergangsregelung ohne formale Absolvierung des Curriculums die Anerkennung der Zusatzqualifikation Sportkardiologie in den Stufen 1, 2 und 3 erhalten, wobei für Stufe 3 zusätzlich die Zusatzbezeichnung Sportmedizin vorliegen muss. Die im Rahmen der Übergangsregelung erworbene Anerkennung hat eine Gültigkeit von 7 Jah- ren. Danach ist eine Rezertifizierung erforderlich.

\section{Korrespondenzadresse}

Prof. Dr. med. C. Burgstahler

Medizinische Klinik, Abteilung Sportmedizin, Universitätsklinikum Tübingen

Hoppe-Seyler-Str. 6, 72076 Tübingen,

Deutschland

christof.burgstahler@med.uni-tuebingen.de

\section{Einhaltung ethischer Richtlinien}

Interessenkonflikt. Den Interessenkonflikt der Autoren finden Sie online auf der DGK-Homepage unter http://leitlinien.dgk.org/ bei der entsprechenden Publikation.

Dieser Beitrag beinhaltet keine von den Autoren durchgeführten Studien an Menschen oder Tieren.

Die Online-Version des Originalartikels ist unter https://doi.org/10.1007/s12181-019-0299-0zu finden. 